\title{
Efficient multi-period distribution mechanism for the innovation investment system under uncertainty
}

\author{
Alim Gurtuev *, Elena Derkach, and Anzor Sabanchiev \\ Kabardino-Balkarian Scientific Center of the Russian Academy of Sciences, Institute for Computer \\ Science and Problems of Regional Management, 360004 Nalchik, Russia.
}

\begin{abstract}
In this paper, we study the problem of a venture investor who distributes the budget between several innovation projects under conditions of uncertainty. A common method for solving this problem is through bilateral negotiations with the external evaluation of projects. However, the effectiveness almost entirely depends on the evaluation quality, but external evaluation seldom reduces the knowledge asymmetry for innovation projects. We propose an iterative revelation mechanism for this problem when the investor sequentially offers possible allocations of the limited budget in the form of threshold dividing questions. The binary choices of innovators serve as a signal of internal estimates of the project implementation costs. Under perfect information, such a mechanism, regardless of the method for determining budget allocations, always produces an effective allocation in subgame-perfect Nash equilibrium. Under uncertainty, the method of offering distribution options matters the optimal solution is found under the English auction class of mechanisms. In an efficient iterative allocation mechanism for innovation investment, the investor proposes a new allocation of the budget each round until an efficient allocation is achieved. The proposed mechanism does not necessarily need to identify the exact minimum budgets for each innovator. Another advantage of the proposed mechanism is the ability to use different processes for organizing rounds.
\end{abstract}

\section{Introduction}

Consider the problem of a venture investor distributing the budget between several innovation projects. The investor cannot directly estimate the cost for implementing each of the projects due to the knowledge asymmetry [1]. At the same time, the investor knows that the budget initially requested by the innovator is excessive and includes optional additional costs and redundant reserves for unexpected events [1]. One of the methods for solving this problem is a series of bilateral negotiations with each of the innovators. During the negotiations, if necessary, an additional external examination of the project is carried out. The disadvantage of this approach is that, with significant additional costs, its effectiveness

\footnotetext{
*Corresponding author: alemao@mail.ru
} 
almost entirely depends on the quality of external expertise. As shown in the literature, for innovation projects, there is a high probability that knowledge asymmetry also exists in the innovator-expert system [1]. Another prominent approach is to use a so-called revelation mechanism when an investor offers innovators a menu of possible solutions. Innovators then send signals about their internal estimates of project implementation costs by choosing specific solutions.

Consider, for example, the situation in the innovation investment model, similar to one described in [1], when an investor with a budget of $B=100$ distributes funding between two innovation projects. Each of the projects in the model has two estimates of the cost of execution - the minimum costs $C_{\min }$ and the premium costs $C_{\text {extra }}$. By definition, the $C_{\min }$ estimate is private information hidden for everyone except the investor herself, and the $C_{\text {extra }}$ estimate is public and acts as the requested budget. Suppose that for project $1 C_{\min }=$ 30, $C_{\text {extra }}=50$, and for project $2 C_{\min }=60$, and $C_{\text {extra }}=90$.

Thus, the investor's budget is less than the total requested budget of both projects. In order to find an efficient budget allocation, the investor offers the innovators some funding as a result of splitting the overall budget $\left(B_{1}, B_{2}\right)$, where $B_{1}$ is the funding share of the first project and $B_{2}$ is the funding share of the second. If both innovators agree with the proposal, the projects are funded. If one of the innovators does not agree, the whole budget is redistributed, moving the split slightly in her favor, and a new proposal is received from the investor. This process continues till one of the innovators agrees on the proposed budget. If at some point it turns out that both disagree, neither of the projects is funded.

Suppose both innovators respond to the proposal truthfully, and the investor starts by proposing an equal budget allocation $\left(B_{1}, B_{2}\right)=(50,50)$. The first innovator agrees because the proposed budget is more than her minimum cost, but the second does not agree. Then the investor proposes a new allocation $\left(B_{1}, B_{2}\right)=(45,55)$, gets the same answers, then proposes $\left(B_{1}, B_{2}\right)=(40,60)$, to which both innovators would agree. Thus, the first project receives funding of 40 , and the second - of 60 .

Indirect revelation mechanisms using dividing menus of "yes/no" type have been relatively well researched and described in the modern literature [2-5]. In [3], in particular, the difference between the costs associated with the definition of distribution rules and the costs associated with the implementation of these rules was investigated, and the existence of additional costs in such mechanisms in determining incentive-compatible payments was shown. In the above example, the investor successfully allocates the budget when the innovators behave truthfully, but the rule itself does not induce incentive compatibility among innovators. The second innovator apparently has incentives to disagree with funding at 60 because it does not include any premium. The investor, however, does not receive any information from the innovators' decisions in order, even ex-post, to form an incentivecompatible budget allocation.

To solve this problem, consider a class of iterative revelation mechanisms for dynamic resource allocation, consisting of the so-called threshold dividing questions and a simple rule for the transfer of funds such as "pay as bid." In each round of such a mechanism, the investor proposes a budget allocation to the innovators. Innovators can agree or disagree but not put forward their proposal (i.e., the possible answers are binary - either "yes" or "no"), and the answer "yes" obliges the innovator to accept the budget in this amount or higher. As a result of iterative conducting of such a survey, the investor determines the prices of innovators and forms the final budget distribution.

Under the condition of complete information in such a mechanism, there is always an effective allocation, in the form of the subgame-perfect Nash equilibrium, regardless of the principle of determining the proposed budget allocations. 


\section{A brief literature review}

The number of works devoted to the design of mechanisms in conditions of high communication complexity has grown very rapidly in the last two decades. Static singlecommodity auction mechanisms with a limited set of messages are considered in [6;7]. In [8], the problems of the theoretical feasibility of the optimal distribution rule taking into account information characteristics in several variants of mechanisms are considered, in [4], necessary and sufficient conditions for Bayesian incentive compatibility are proposed, in [9], the design of an iterative revealing mechanism for a single-commodity auction with limited communication.

This paper considers a multi-agent system in which agents (innovators) have quasilinear utility functions and belong to one of the disjoint types. Every innovator has dichotomous preferences, i.e., the results of the mechanism work fall into one of two classes - "good" or "bad." Moreover, each innovator equally evaluates each result of a particular class. Indeed, in the example discussed earlier, it makes no difference to determine the answer of the first innovator whether the investor proposes the distribution $(40,60)$ or $(50,50)$ - in both cases, the answer of the innovator will be positive. Thus, the type of agent can be represented by one value - the assessment of a "good" result, taking the assessment of "bad" as zero.

A similar approach to normalization estimates is widely used in the literature $[5 ; 10]$. It allows, with small assumptions, to consider both the mechanisms for organizing the distribution of funding from one source and several competing sources simultaneously [1112]. With the simultaneous existence of several competing proposals, it is difficult to identify market participants' preferences since both for innovators and investors, in this case, the utility function becomes more complicated than in the one source case. In such cases, Vickrey-Clarke-Groves (VCG) mechanisms are effective, which are ex-post compatible in preference [13-15]. Note that in this case, the number of interactions (message exchanges) required to achieve the optimal distribution grows exponentially with the increase in the number of proposals [15].

\section{Model}

Let $N$ be the set of all innovators receiving venture funding. The venture investor chooses some budget distribution $x$ from a finite set of such distributions $X$. Each innovator is characterized by a quasilinear utility function $u_{\mathrm{i}}: X \rightarrow Z$ and has dichotomous preferences. Thus, the function of evaluating the innovator takes the form

$$
u_{i}(x)= \begin{cases}v_{i}, & \text { if } x \in X_{i} \\ 0, & \text { if } x \notin X_{i}\end{cases}
$$

Let us assume that $v_{i}$ belongs to some set of possible estimates $V$, bounded above and below, and known to all participants in the system. For each specific innovator, $v$ coincides with his minimum cost $C_{\min }$.

The investor's goal in such a system is to find an efficient budget allocation

$$
x^{*}(v) \in \operatorname{argmax}_{x \in X} \sum_{i \in N} u_{i}(x)
$$

where $v=\left(v_{i}, \ldots, v_{i}\right)-$ vector of evaluations of innovators. 
For simplicity, we assume that the range of values of the utility functions is the set of real numbers and that for any state of the system, there is a unique optimal solution to the distribution problem.

The total public utility function $\mathrm{W}(\mathrm{u})$ in the state of the system $\mathrm{v}$ is determined by the maximum utility of the participants in the system with optimal allocation:

$$
W(v)=\max _{X} \sum_{N} u_{i}(x)
$$

In a similar way, it is possible to determine the function of total public utility for a system without a specific participant $\mathrm{i}$ :

$$
W_{-i}\left(v_{-i}\right)=W\left(\underline{v}_{i}, v_{-i}\right)
$$

\section{Results}

In the system of innovation investment, when investors and innovators interact, naturally, the parties cannot observe the actual values of each other's assessments - neither the innovators know the size of the investor's budget, nor can the investor distinguish the minimum $C_{\min }$ costs and $C_{\text {extra }}$ premium costs for each project. To achieve a rational distribution of the investment budget, an investor in such a system gradually collects information about innovators through sequential binary questions. This procedure is structured as follows: the investor simultaneously offers a specific budget to all innovators. When an investor proposes a budget $p_{i}$, and innovator $i$ accepts it, this means that $v_{i} \leq p_{i}$, that is, the minimum budget required for an innovator, is no more than the investor's proposal. Step by step, by proposing different budget allocations, the investor determines the limits of $v_{i}$ for all innovators. For example, the fact that the first innovator refuses the distribution $(40, \ldots)$, but accepts the distribution $(70, \ldots)$ means that his minimum cost $\boldsymbol{C}_{\boldsymbol{m i n}}$ lies within the boundaries $[40,70]$.

We define an iterative allocation mechanism (IAM) as a multi-period non-cooperative game as follows:

$$
\Gamma=\left(\left\{J^{\mathrm{t}}, p^{\mathrm{t}}\right\}_{\mathrm{t}}, g, p\right)
$$

Here $t$ is the round of the game, $J_{t}$ is the set of innovators to whom the investor proposes a budget allocation, while $h^{t} \in H^{t}$ denotes the history of the game at the end of round $t$. The budget offered to innovator $i$ is defined as $p_{i}^{t}: H^{t-1}$. Without loss of generality, we can assume that for every round $t$, every history $h^{t-1} \in H^{t-1}$, and every rational innovator $i \in J^{t}\left(h^{t-1}\right), p_{i}^{t} \in V_{i}\left(h^{t-1}\right) \backslash\left\{\underline{v}_{i}\left(h^{t-1}\right)\right\}$. Each innovator at time $t$ makes a decision on participation in the budget allocation \{“yes", "no"\}. The game stops at time $T$ when $J^{T+1}\left(h^{\tau}\right)=0$. Thus, the process $\left\{J^{t}, p^{t}\right\}_{t}$ describes a game tree in which each node generates two subsequent nodes, and $g$ and $p$ are functions that map each end node (leaf) of this tree to some budget distribution.

All participants of the mechanism - both the investor and the innovators, know all the information about the previous rounds. The equilibrium in such a mechanism is the subgame-optimal Nash equilibrium. Suppose the communication between the participants of the mechanism takes place in the cheap talk mode, i.e. without transaction costs, and there are no restrictions on the number of game rounds or its duration. In that case, the investor proposes as many sequential budget allocations as needed until an efficient allocation would be found. 
An iterative allocating mechanism leads to an efficient allocation for a specific history $h$ if there is a distribution $x_{h} \in X$ and $x^{*}(\tilde{v})=x_{h}$ for all $\tilde{v} \in V(h)$. Let us call an allocation mechanism efficient if it always leads to an efficient allocation while all participants act in accordance with their true preferences.

In an efficient iterative allocation mechanism for innovation investment, the investor must propose a new allocation of the budget each round until an efficient allocation would be achieved. At the same time, the proposed mechanism does not necessarily need to identify the exact minimum cost $C_{\min }$ of each innovator. Another advantage of the proposed mechanism is the possibility of using various processes for organizing rounds - these can be auction mechanisms with an increasing price, with a decreasing price, with an increase in the number of innovators, et cetera.

\section{Discussion}

It is easy to see that in a set of economic systems with dichotomous agent preferences and costly signals [16], the aggregate utility function for a coalition of agents may not be submodular. Submodularity presupposes the fulfillment of the substitutes condition, while dichotomous preferences, on the contrary, most often exhibit the property of complementarity. For example, this is typical for the problem of financing a public good, where the marginal contribution of each agent does not decrease with the size of the economic system. For such a system, subject to costly signals, it is impossible to create a mechanism in the form of an English auction with a VCG result [17]. Thus, it is also impossible to construct an efficient iterative allocation mechanism for such systems.

The assumption of cheap talk property dramatically facilitates the creation of our mechanism, but there are still systems for which it is impossible to achieve efficient ex-post equilibrium [3]. The iterative allocation mechanism may not gather enough information for these systems to calculate the VCG result, but an effective ex-post equilibrium assumes a VCG-equivalent mechanism. At the same time, it is known that each effective allocating rule is realized as a Bayes-Nash equilibrium [3].

At the same time, for many systems, particularly for the system of innovation investment, an iterative distribution mechanism can be created, and it allows various forms of implementation, depending on the specific system.

\section{Conclusion}

For a number of economic systems, particularly for the system of financing innovation projects, an iterative allocation mechanism is an effective mechanism for allocating budgets in the face of uncertainty and hidden preferences of agents. The iterative allocation mechanism described in this article belongs to the class of dynamic indirect revelation mechanisms. In this mechanism, the budget allocating investor iteratively proposes allocation options to innovators and determines the efficient allocation after a series of such offers.

With complete information and dichotomous revealed preferences, the effective allocation is the subgame-optimal Nash equilibrium, regardless of the method of proposing distribution options. Under the condition of uncertainty, the method of offering distribution options matters - the optimal solution is found when it is organized in one of the forms of the English auction. However, an iterative allocating mechanism does not necessarily exist for each specific system with independent agents that are incentive-inconsistent.

The research was supported by the Russian Foundation of Basic Research, grant No. 19-010-00376A

"Mechanisms for screening innovation projects on the basis of the analysis of tacit knowledge." 


\section{References}

1. A. Gurtuev, E. Derkach, S. Makhosheva, Z. Ivanov, Heliyon, 6, 11 (2020)

2. T. van Zandt, J. Eur. Econ. Association, 5, 2/3 (2007)

3. R. Fadel, I. Segal, J. Econ. Theory, 144, 5 (2009)

4. D. Mookherjee, M. Tsumagari, J. Polit. Econ., 122, 5 (2014)

5. R. Sano, KIER WP, Kyoto University, 937 (2016)

6. L. Blumrosen, N.Nisan, I.Segal, J. Artificial Intelligence Research, 28 (2007)

7. N. Kos, Games and Econ. Behavior, 75 (2012)

8. L. Blumrosen, M. Feldman, Games and Econ. Behavior, 82 (2013)

9. N. Kos, Games and Econ. Behavior, 87 (2014)

10. D. Mishra, S. Roy, Theoretical Econ, 8 (2013)

11. D. Lehmann, L.I. O'Callaghan, Y.Shoam, J. of ACM, 49 (2002);

12. P. Milgrom, I. Segal, The Deferred-Acceptance Heuristic Auctions (Stanford University, 2014)

13. L. Ausubel, P. Milgrom, Frontier of Theoretical Econ., 1 (2002)

14. L. Ausubel, American Econ. Review, 96 (2006)

15. N. Nisan, I. Segal, J. Econ. Theory, 129 (2006)

16. A. Muramoto, R. Sano, Econ. Letters, 149, (2016)

17. S. de Vries, J. Schummer, R.Vohra, J. Econ. Theory, 132 (2007) 